\title{
Historia del manejo de rinitis atrófica
}

\author{
The history of the treatment of atrophic rhinitis
}

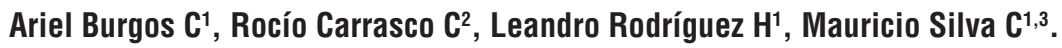

\section{RESUMEN}

La rinitis atrófica es una enfermedad crónica progresiva caracterizada por dilatación anormal de las cavidades nasales con atrofia de la mucosa, submucosa y cornetes nasales subyacentes. Los factores etiopatogénicos aún son desconocidos. Su presentación clínica consiste en congestión nasal paradójica asociado a secreciones viscosas, con presencia de costras secas de mal olor. La higiene nasal con irrigación de alto volumen y baja presión es el estándar de tratamiento médico. El tratamiento quirúrgico busca reducir el tamaño de las cavidades nasales y promover la regeneración de la mucosa nasal así como también su vascularización y lubricación. A lo largo de la historia se han descrito múltiples procedimientos quirúrgicos que han buscado estrechar la cavidad nasal para permitir el paso de aire de forma más fisiológica. Por otra parte, se han propuesto intervenciones radicales como el cierre de las fosas nasales para disminuir los síntomas y mejorar la calidad de vida. En este artículo se resumen los principales manejos y procedimientos propuestos junto con sus resultados y conclusiones. Si bien la mayoría de las técnicas descritas ya no se utilizan en la actualidad, es importante conocerlas ya que aún existen pacientes que fueron sometidos a ellas pudiendo presentar complicaciones y/o efectos adversos.

Palabras clave: Rinitis atrófica, ozena, rinitis sicca.

\begin{abstract}
Atrophic rhinitis is a chronic progressive disease characterized by abnormal dilatation of the nasal cavities with atrophy of the mucosa, nasal submucosa and underlying nasal turbinates. The etiopathogenic factors are still unknown. Its clinical presentation consists of paradoxical nasal congestion associated with viscous secretions, usually with the presence of dry, bad-smelling crusts. Nasal hygiene with high pressure irrigation remains the standard of medical treatment. Surgical treatment seeks to reduce the size of nasal cavities and promote regeneration of nasal mucosa as well as its vascularization and lubrication. Throughout history, multiple surgical procedures have been
\end{abstract}

\footnotetext{
1 Departamento Otorrinolaringología, Hospital del Salvador, Universidad de Chile, Santiago, Chile.

2 Escuela de Medicina, Facultad de Medicina, Universidad de Chile, Santiago, Chile.

3 Servicio de Otorrinolaringología, Hospital Del Salvador, Universidad de Chile, Santiago, Chile.

Los autores declaran no tener conflicto de interés.
}

Recibido el 14 de octubre de 2019. Aceptado el 11 de diciembre de 2019. 
described that have sought the narrowing of the nasal cavity to allow the passage of air more physiologically. On the other hand, radical interventions have been proposed such as the closure of the nostrils to reduce symptoms and improve quality. This article summarizes the main proposed procedures along with their results and conclusions. Although most of the techniques described are no longer used today, it is important to know them since there are still patients who were subjected to them and may present complications and / or adverse effects.

Key words: Atrophic rhinitis, ozena, sicca rhinitis.

\section{INTRODUCCIÓN}

La rinitis atrófica, rinitis sicca o también denominada ozena, fue descrita por primera vez en el papiro de Edwin (1700 aC.), donde los egipcios sugerían un tratamiento con vino y leche materna. Más de 3000 años después, en 1879, Fraenkel caracterizó una tríada de síntomas que consistía en mal olor nasal, atrofia de la mucosa y formación de costras'. Actualmente, se define como una enfermedad crónica progresiva caracterizada por dilatación anormal de las cavidades nasales con atrofia de la mucosa, submucosa y de los cornetes nasales subyacentes².

Los factores etiológicos que participan en su patogenia son aún desconocidos. Sin embargo, se conoce que ocurren una serie de cambios histopatológicos tales como la pérdida precoz del epitelio ciliado columnar con presencia de áreas de metaplasia escamosa e inflamación crónica que lleva a fibrosis de los tejidos. A nivel vascular, los fenómenos observados permiten clasificarla en dos tipos. La tipo I es la más frecuente, correspondiendo al $50 \%-80 \%$ de los casos y se caracteriza por la presencia de fenómenos inflamatorios, tales como endarteritis obliterante, periarteritis y fibrosis de arteriolas terminales. La tipo II es menos común (20\%-50\% de los casos) y es aquella que presenta vasodilatación capilar. En ambos tipos, los cambios tisulares mencionados llevan a una alteración del clearance mucociliar y con ello a una estasis de las secreciones nasales y formación de costras ${ }^{3}$.

Uno de los factores más demostrados que participan en la patogenia de esta enfermedad corresponde a las alteraciones autonómicas, fundamentalmente la vasoconstricción excesiva. De este modo, algunos autores postulan que tanto la rinitis atrófica primaria como la rinitis vasomotora forman parte del mismo espectro de patologías 4 .
Las infecciones bacterianas crónicas han sido descritas como parte de la patogenia de la rinitis atrófica primaria. Entre los microorganismos asociados destaca Klebsiella ozaenae, Coccobacillus foetidus ozaena, Haemophilus influenzae, Pseudomona aeruginosa, Proteus spp, entre otros. Si bien existe escasa evidencia de que estos agentes son la causa primaria de la enfermedad, se ha postulado que la infección con dicha flora mixta, generaría ciliostasis y con ello destrucción epitelial4.

Estudios recientes, sugieren que en la rinitis atrófica existe una alteración de la geometría de la cavidad nasal, así como también un flujo de aire no fisiológico debido a la pérdida anormal de agua en la mucosa. En pacientes con rinitis atrófica, el área transversal de la cavidad nasal es considerablemente mayor que en personas sanas, con una discreta disminución a nivel de la válvula nasal. Mientras que en las personas sanas la humidificación del aire ocurre preferentemente en la porción anteroinferior de la cavidad nasal, en el paciente con rinitis atrófica este flujo está aumentado de forma generalizada, especialmente en la porción superior de la cavidad nasal y en la porción proximal del cornete medio, lo cual se correlaciona con las áreas de mayor formación de costras ${ }^{5}$.

La edad de presentación es más frecuente en adultos jóvenes o de edad media de la vida, siendo rara antes de la pubertad. En algunas series se ha mencionado su mayor prevalencia en mujeres que en hombres con una relación 5,6:1. Autores han planteado la asociación de esta patología con alteraciones hormonales. Lo anterior se sustenta en el inicio después de la pubertad, el empeoramiento de los síntomas durante la menstruación y el embarazo, y la mejoría de las molestias en algunos casos de mujeres sometidas a tratamientos con estrógenos 4 . En el último siglo se ha observado una disminución importante en la 
incidencia de rinitis atrófica, debido posiblemente al uso de antibioterapia en el tratamiento de infecciones nasales crónicas. Su incidencia actual es mayor en países de climas cálidos y va desde 0,3\% a 7,8\% en lugares como India, China, Egipto, Pakistán, Filipinas, Malasia, Arabia Saudita, centro de África, entre otros ${ }^{2}$. Si bien se reconoce como una enfermedad de origen multifactorial y posible herencia poligénica, en algunos estudios se ha observado que el $27,4 \%$ de los casos son hereditarios, con un patrón de herencia autosómico dominante en el $67 \%$ de ellos y autosómico recesivo en el restante. En otros estudios se ha reportado que existe el antecedente familiar en el $15 \%-30 \%$ de los casos ${ }^{4}$.

Desde el punto de vista clínico, su presentación consiste en la presencia de secreciones nasales viscosas, congestión nasal paradójica y costras secas de mal olor, lo que se denomina ozena $^{2}$, término que proviene del griego "ozaina" que significa hedor. Los síntomas asociados incluyen anosmia, sequedad nasal, obstrucción nasal, epistaxis episódica y, en algunos casos, perforación del tabique nasal ${ }^{6}$. Ruskin en 1932, clasificó esta entidad en dos tipos, primaria y secundaria ${ }^{1}$. La primaria corresponde a un cuadro de etiología no especificada, de inicio espontáneo y progresión lenta en el tiempo. Por su parte, la secundaria es aquella que se desarrolla después de traumatismos nasales 0 sinusales, cirugías reductoras nasales, radioterapia 0 rinosinusitis crónica asociadas a enfermedades granulomatosas crónicas de la nariz². Si bien el uso de cocaína, corticosteroides locales o descongestionantes intranasales por periodos prolongados y el trauma digital pueden estar implicados en el desarrollo de perforación del tabique nasal, generalmente no se asocian con rinitis atrófica ${ }^{6}$.

Considerando la sintomatología poco específica de la rinitis atrófica sumado a la multiplicidad de entidades asociadas a su manifestación secundaria, se hace indispensable conocer sus diagnósticos diferenciales. Entre ellos destaca la presencia de cuerpos extraños endonasales 0 rinolitos, infección crónica rinosinusal unilateral y la supuración adenoidea en los adolescentes ${ }^{4}$. Otro concepto que puede confundirse con rinitis atrófica es el llamado síndrome de nariz vacía, entidad de origen exclusivamente iatrogénico que se genera posterior a una turbinectomía y que se diferencia de la rinitis atrófica por la ausencia de mal olor y de patógenos asociados ${ }^{7}$.

En cuanto al examen endoscópico en rinitis atrófica, es posible encontrar en muchos casos ausencia total o parcial de los cornetes inferiores $\mathrm{y} / 0$ medios $^{8}$. Lo anterior concuerda con un estudio retrospectivo realizado en 242 pacientes con rinitis atrófica secundaria en que se observó ausencia parcial del cornete inferior en el $62 \%$ de ellos y ausencia total en el $37 \%$. Por otra parte, se encontró ausencia del cornete medio en el $57 \%$ de los pacientes y en el $32 \%$ de ellos existía solo un remanente ${ }^{8}$. Desde el punto de vista imagenológico, en la tomografía computarizada de senos paranasales se puede observar cavidades nasales amplias, engrosamiento de la mucosa, pérdida de la definición del complejo ostiomeatal y destrucción ósea del cornete inferior y medio ${ }^{8}$.

\section{Tratamiento médico}

Como múltiples estudios han evidenciado, el tratamiento de la rinitis atrófica es tan controvertido como variado ${ }^{8}$. El manejo médico estándar consiste en higiene nasal a través de irrigación de alto volumen y baja presión, ya sea con soluciones salinas isotónicas, hipotónicas 0 hipertónicas. Numerosos estudios han demostrado que la aplicación diaria de soluciones isotónicas favorece la prevención y el tratamiento de infecciones rinosinusales sin efectos adversos relevantes ${ }^{9}$. También se ha reportado el uso de soluciones de glucosa y glicerina administradas tres 0 cuatro veces al día. La glucosa al 25\% actuaría acidificando las cavidades nasales y con ello inhibiría la infección saprofítica y de bacterias proteolíticas, promoviendo el crecimiento de flora comensal. La glicerina por su parte, favorecería la lubricación de las fosas nasales impidiendo la formación de costras ${ }^{4}$. No obstante, no hay estudios que muestren evidencia suficiente respecto al empleo de estas soluciones. Por otra parte, se ha descrito la aplicación de parafina líquida con el objetivo de lubricar la mucosa nasal y favorecer la eliminación de costras. Sin embargo, no se recomienda su uso a largo plazo debido a su asociación con la aparición de granulomas de parafina y neumonía lipoídea ${ }^{4}$. 
A pesar del amplio uso de ungüentos nasales con el objetivo de lubricar la mucosa nasal y reducir la pérdida de agua, no hay evidencia que respalde su aplicación en pacientes con rinitis atrófica?. Otros aspectos fundamentales en el tratamiento médico son la remoción de costras por parte del especialista y la educación del paciente respecto de su patología. Adicionalmente se pueden utilizar antibióticos para el control de las exacerbaciones ${ }^{2}$. En una revisión realizada por Hildenbrand el año 2010, se sugiere el uso de antibióticos tales como aminoglucósidos, rifampicina $600 \mathrm{mg}$ una vez al día durante 12 semanas o ciprofloxacino 500 a $750 \mathrm{mg}$ dos veces al día durante 8 a 12 semanas $^{9}$. Respecto a la toma de cultivos, no existe evidencia disponible acerca de su utilidad y momento de obtención en rinitis atrófica ${ }^{10}$.

\section{Tratamiento quirúrgico}

A lo largo de la historia se han descrito múltiples procedimientos quirúrgicos que han buscado permitir el paso de aire de forma más fisiológica. Algunos de ellos se han propuesto estrechar la cavidad nasal basándose en que al disminuir el flujo de aire durante la inspiración, disminuye la sequedad nasal y con ello la formación de costras. Por otra parte, se han descrito intervenciones más radicales como lo es el cierre de las fosas nasales buscando disminuir los síntomas y mejorar la calidad de vida de los pacientes ${ }^{10}$. A continuación se resumen los principales procedimientos y manejos postulados junto con sus resultados y conclusiones.

\section{Cierre de fosas nasales}

Con el objetivo de facilitar la regeneración de la mucosa nasal, en 1967 Young describió el cierre bilateral de las fosas nasales en 11 pacientes. El procedimiento consistía en el levantamiento y posterior sutura de colgajos dentro de una fosa nasal. En un segundo tiempo quirúrgico se obliteraba la segunda fosa y se realizaba una revisión de la primera que incluía toma de muestras para biopsia. En el artículo original se describen resultados alentadores, con desaparición de los síntomas y el hallazgo de mucosa nasal aparentemente normal en 6 de ellos. No hubo recurrencia de síntomas en el seguimiento de uno a dos años. En el mismo estudio se detalla la implantación de diversos materiales tales como hueso, esponjas plásticas y polietileno en la submucosa nasal, sin embargo, no se menciona la técnica quirúrgica ni los eventos adversos asociados ${ }^{12}$.

Años después (1971), un grupo de estudio en India replicó la técnica quirúrgica en 37 pacientes, realizando cierre bilateral en 17 y unilateral en 20 de ellos. En estos últimos se describe una disminución de los síntomas y costras nasales en el primer mes posoperatorio. En los casos de cierre bilateral también se reporta desaparición de los síntomas dentro del primer mes y buena tolerancia a la respiración bucal. Con respecto a los efectos adversos, se informó sequedad bucal al despertar, lo que fue resuelto a través del aumento de la ingesta de agua en la mayoría de los pacientes. Por otra parte, un grupo de los afectados refirieron ronquidos nocturnos, pero no se evaluó la significancia de éstos. Dos casos con cierre bilateral tuvieron que reabrirse, una adolescente de 17 años después de ocho meses de cierre, por razones maritales y un hombre de 60 años luego de dos meses de cierre, ya que no logró tolerar la respiración bucal. Lo anterior permitió la revisión de las fosas nasales, encontrándose mucosa húmeda y rosada, sin costras, pero con persistencia de la atrofia de cornetes ${ }^{13}$.

En relación a la reapertura de las fosas nasales ninguno de los estudios llega a un consenso, sin embargo, el segundo plantea su realización luego de tres años en el caso de cierre bilateral y cinco años en los unilaterales ${ }^{13}$. En el año 2012, una revisión sistemática Cochrane, señaló que existían importantes deficiencias metodológicas en los estudios mencionados, lo que limita la validez de los hallazgos ${ }^{10}$. Posteriormente se publicó la modificación del procedimiento de Young, que consistía en el cierre parcial de la fosa nasal dejándola de un diámetro de $3 \mathrm{~mm} 0$ menos. Esta intervención reportaba la ventaja de ser realizada en ambas fosas nasales en un mismo tiempo quirúrgico, permitiendo además la posibilidad de realizar examen físico y revisión endoscópica seriada. De igual forma se aseguraba que los pacientes mantenían en parte la respiración nasal, con ausencia de hipernasalidad y un mejor resultado estético. No obstante, generaba molestias respiratorias y alteraciones del olfato en 
Ios pacientes. Con esta cirugía no se reportaron casos de recurrencia en 2 años de seguimiento ${ }^{12}$.

En 1988, investigadores de Arabia Saudita estudiaron el efecto a nivel histológico tras el cierre quirúrgico parcial de la fosa nasal en 20 pacientes jóvenes (18 a 32 años) que padecían rinitis atrófica. A nivel epitelial se observó recuperación del aspecto pseudoestratificado, con estrechamiento de los espacios intercelulares y células con abundante citoplasma y límites regulares. A nivel vascular, se observó la reapertura de arteriolas y capilares, no observándose cambios en estroma circundante. En resumen, concluyeron que la operación tenía un efecto beneficioso sobre la mucosa nasal siendo capaz de revertir las alteraciones patológicas a nivel microanatómico ${ }^{14}$. En el año 2001 se publicó un estudio que planteaba nuevos cambios en la técnica quirúrgica de la cirugía de Young modificada aplicados en un total de 20 pacientes. La técnica descrita en dicha publicación, consistía básicamente en la confección de un colgajo a partir de la pared lateral nasal, el cual se replegaba sobre sí mismo a través de una sutura, generando finalmente un cierre parcial de la fosa nasa $\left.\right|^{15}$. La evaluación de los resultados poscirugía se realizó considerando la disminución de síntomas y signos, así como también los cambios histopatológicos en la pared nasal lateral a los 6 meses. El $50 \%$ de los pacientes presentó recuperación completa, 40\% recuperación parcial y el 10\% no presentó mejoría. Como ventajas del procedimiento se menciona la simpleza de la técnica en comparación con intervenciones preexistentes, minimización de la dificultad respiratoria, posibilidad de examen físico regular, limpieza de la cavidad nasal y posibilidad de realizarse de forma simultánea en ambas fosas nasales ${ }^{15}$.

\section{Trasplante de mucosa del seno maxilar}

Con el objetivo de mejorar la lubricación de la mucosa nasal en 1978 Raghaw Sharan postuló la realización de un trasplante de mucosa del seno maxilar a la cavidad nasal en forma de bolsa evaginada. El procedimiento tenía como objetivo no solo disminuir el tamaño de la cavidad nasal sino que también aumentar la secreción nasal y favorecer su lubricación. El procedimiento consistía en realizar un abordaje sublabial, tras lo cual se procedía a separar la mucosa del seno maxilar del hueso subyacente excepto la mucosa cercana al ostium. Posteriormente la bolsa de mucosa formada se evaginaba a través del ostium, dejándola dentro de la cavidad nasal y suturándola a la pared medial y lateral. La intervención se llevó a cabo en 7 pacientes con el diagnóstico de rinitis atrófica. Se realizó seguimiento de los pacientes a los tres meses, seis meses y un año posterior a la cirugía y la evaluación de los resultados fue considerando sintomatología y cambios histológicos. Se observó desaparición de los síntomas en 4 pacientes $(55,5 \%$ del total) a 1 ó 2 meses después de la intervención quirúrgica. No sólo desaparecieron las costras y el mal olor nasal sino que también la mucosa nasal se volvió húmeda y rosada. La anosmia también reportó mejoría. En 2 casos (28,5\% del total) los síntomas se prolongaron por más de 6 meses posterior a la cirugía. En éstos últimos, hubo una mejoría parcial con desaparición o disminución de la cacosmia, pero persistencia de costras y anosmia. Particularmente en uno de esos 2 casos, hubo descarga mucopurulenta posoperatoria que desapareció luego de un mes. En el paciente restante, si bien hubo mejoría inicial, después de siete meses los síntomas de rinitis atrófica reaparecieron. La justificación de este fracaso terapéutico fue la presencia de una mucosa antral muy atrófica, asociada a una patología de larga data, que durante la cirugía se desgarró en varios puntos, impidiendo la eversión de una bolsa de mucosa en forma adecuada. Desde el punto de vista clínico e imagenológico no hubo efectos adversos secundarios en los senos maxilares. Con respecto a la histología, es importante mencionar que se realizó biopsia preoperatoria en los 7 casos, observándose epitelio escamoso estratificado con escasas glándulas mucosas y presencia de células inflamatorias. A un año de la cirugía las biopsias mostraron normalización del revestimiento epitelial de la cavidad nasal. En casos donde persistió el epitelio escamoso hubo restauración de las glándulas mucosas, excepto en un caso donde no se observaron cambios. Finalmente, el autor concluyó que en pacientes con rinitis atrófica de poco tiempo de evolución y mucosa sinusal normal, el trasplante de mucosa del seno maxilar tiene resultados alentadores y duraderos ${ }^{16}$. 


\section{Implante de materiales artificiales y biológicos}

El implante artificial de diversos materiales surgió con el objetivo de restaurar el volumen intranasal, siendo realizado desde inicios del siglo pasado cuando Gersuny inyectó parafina bajo la mucosa nasal ${ }^{7}$. Posteriormente, a mediados de siglo, el implante de materiales se realizó en conjunto con las primeras cirugías de cierre de fosas nasales. Sin embargo, en dichos estudios no se menciona la composición de los implantes ni tampoco la técnica quirúrgica con la que eran insertados. Figura 1, muestra ejemplo de material sintético utilizado en paciente sometido a cirugía de rinitis atrófica en la década de 1950.

En 1947, Proud estudió la inserción de implantes septales de metacrilato de metilo en 7 pacientes. Estos se insertaban entre colgajos de mucosa que posteriormente se suturaban. En 6 casos se redujo de manera importante la formación de costras nasales, mientras que en un paciente el resultado solo fue parcial, debido a que los implan- tes eran muy pequeños para la cavidad nasal. Por otra parte, 2 de los 3 pacientes que habían perdido el olfato, lo recuperaron después de la intervención. Sólo un paciente no presentó mejoría, lo que fue asociado a una técnica quirúrgica defectuosa y posterior extrusión de los implantes. Para solucionar esto último, se propuso la eliminación de los cartílagos septales, que se encontraban yuxtapuestos al implante. Finalmente, el autor concluyó que la resina acrílica era un material ideal para ser incluido en las fosas nasales, siendo bien tolerada por los pacientes ${ }^{17}$.

Saunders en 1958 puso a prueba la inserción subperióstica en la pared nasal lateral de implantes de hueso ilíaco para el manejo quirúrgico de 8 pacientes con rinitis atrófica. Bajo anestesia local o general se procedía a obtener un injerto óseo, preferentemente de hueso ilíaco esponjoso. Posteriormente se realizaba una incisión en el pliegue nasolabial o vía intraoral a través del surco sublabial. Tomando como referencia la cresta piriforme, se procedía a separar el periostio de la pared nasal

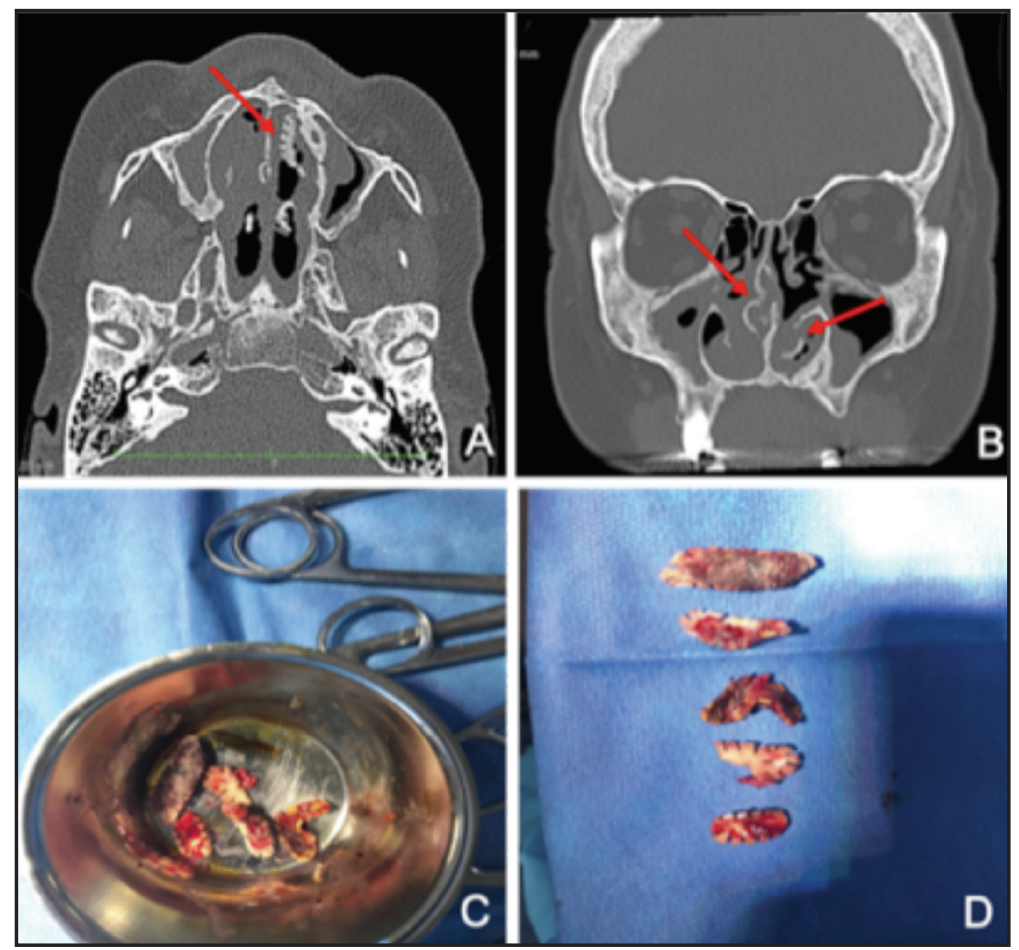

Figura 1. Tomografía computarizada (a) axial y (b) coronal de paciente sometido a cirugía de rinitis atrófica en la década de 1950. Se observan imágenes hiperdensas compatibles con cuerpos extraños en tabique nasal y piso de fosa nasal izquierda (flechas rojas). (c) y (d) cuerpos extraños luego de su extracción por vía endoscópica. 
lateral, tras lo cual se introducían pequeños trozos de hueso esponjoso. En los resultados se menciona una evolución satisfactoria en la sintomatología en 7 pacientes durante un período de seguimiento de tres años. Sólo un paciente no mostró mejoría. El autor postuló dos razones para el fracaso terapéutico: uso de hueso insuficiente 0 absorción del injerto. Por otra parte, 3 pacientes continuaron presentando costras, pero en menor cantidad a lo observado previo a la cirugía. Ninguno de los pacientes presentó epistaxis, costras purulentas ni mal olor ${ }^{18}$.

En 1977 en India, se publicó un estudio realizado en un grupo de 60 pacientes a los que se les implantó una resina acrílica (methyl halacylate) tanto en el septo como en el piso de la fosa nasal, siguiendo la técnica de Proud. Por el contrario, a los resultados encontrados por este último, los implantes mostraron no ser duraderos en el tiempo. Del total de pacientes $81 \%$ mejoró con este tratamiento, pero en el seguimiento realizado dos años después, hubo recurrencia en el $80 \%$ de los pacientes por extrusión del implante y perforación del tabique nasal ${ }^{12}$. En el mismo estudio se describe la instalación de dermo-fat en 4 pacientes. El material biológico era obtenido de la región frontal del muslo de los pacientes y posteriormente implantado en el piso de la nariz a través de un abordaje sublabial. De los sujetos de estudio, en uno se logró eliminación de los síntomas, otro presentó disminución de molestias, mientras que los 2 restantes no presentaron mejoría. No se observó rechazo de los injertos. La contracción y absorción de los injertos fueron reconocidos como los principales factores de fracaso de la intervención. Finalmente se menciona que hubo $50 \%$ de recurrencia de la enfermedad, pero no se especifica en qué tiempo ocurrió. Se concluyó que se trataba de un procedimiento quirúrgico complejo asociado a una hospitalización prolongada y que además no tenía buenos resultados ${ }^{12}$. Posteriormente, se estudió el efecto de inyecciones intranasales de extracto de placenta en 60 pacientes. Consistía en la introducción de 0,5 cc en la submucosa del cornete inferior y medio en cada fosa nasal con intervalos semanales. Los resultados iniciales fueron alentadores, con un reporte de mejoría de los síntomas en $93,3 \%$ de los casos y solo $6,6 \%$ de recurrencia a dos años de seguimiento ${ }^{19}$.
En el año 2000, Goldenberg describió la instalación de plastipore, una esponja de polietileno de alta densidad con microporos que permite el crecimiento del tejido, anclando los implantes al tejido circundante. Se caracteriza por ser un material sintético, biocompatible, elástico, moldeable, resistente a infecciones y que provoca una mínima reacción de cuerpo extraño. Ocho pacientes fueron sometidos al procedimiento, instalándose 2 placas de plastipore de $5 \mathrm{~mm}$ de grosor, $8 \mathrm{~mm}$ de ancho y 4 a $5 \mathrm{~cm}$ de largo, en submucosa de piso y tabique nasal. Se obtuvieron excelentes resultados en 6 pacientes, con resolución completa de los síntomas a 18 meses de seguimiento. En 2 pacientes los resultados fueron buenos presentando solo costras residuales. Un implante se extruyó espontáneamente y se reimplantó. No hubo casos de infección asociados ni otras complicaciones ${ }^{20}$. Dichos estudios, destacan la simpleza de la técnica y la posibilidad de usar anestesia local. Entre sus desventajas se encuentra la extrusión en 1 de 8 pacientes y la necesidad de utilizar mucosa septal de buena calidad para disminuir el riesgo de extrusión tras el procedimiento ${ }^{19}$. En relación con el tipo de material utilizado, Houser en el año 2007, propuso que los mejores resultados se obtenían con aloinjertos tales como costilla, cartílago o dermis acelular. Siguiendo esta lógica, se implantó dermis acelular en ocho pacientes, logrando una mejoría estadísticamente significativa en las puntuaciones de los síntomas medidos a través de SNOT 20 en todos los pacientes después de un período de seguimiento de al menos tres meses ${ }^{20}$. Un año más tarde, Jaswal publicó un estudio cuyo objetivo fue comparar la efectividad del tratamiento con rifampicina oral versus la inyección submucosa de extracto placentario en 30 pacientes con diagnóstico de rinitis atrófica primaria, divididos de forma randomizada en tres grupos de 10 pacientes. Al primer grupo actuó como control por lo que sólo recibió irrigación con solución alcalina compuesta por agua tibia, bicarbonato de sodio, sal y azúcar, una vez al día por tres meses. Al segundo grupo se le inyectó extracto placentario en la submucosa nasal (cada ml de solución contenía 0,1 gr de placenta humana) una vez a la semana por tres meses junto con irrigación nasal con solución alcalina. Finalmente, al tercer grupo se le administró rifampicina en dosis de $10 \mathrm{mg} / \mathrm{kg}$ vía oral por tres me- 
ses e irrigación nasal con una solución alcalina ${ }^{21}$. Los resultados fueron evaluados considerando las modificaciones histopatológicas, la mejoría clínica y los cambios a nivel endoscópico. En relación con el ámbito histológico, se compararon biopsias del tercio medio del cornete inferior obtenidas al inicio del estudio y luego de administrada cada terapia y se observó que el tratamiento con rifampicina favorecía el paso desde un epitelio escamoso a una mucosa nasal normal. En cuanto a la mejoría clínica, los pacientes tratados con rifampicina estuvieron libres de signos y síntomas a los 6 meses de completar la terapia. Al año de seguimiento el $70 \%$ mostró los mismos resultados mientras que el $30 \%$ refirió solo presencia de costras, pero en menor cantidad. Los pacientes tratados con inyecciones de extracto placentario mostraron un intervalo libre de síntomas de 2,7 meses en promedio, con una recurrencia más rápida de los síntomas, siendo las costras el primer síntoma en reaparecer. Los pacientes tratados sólo con irrigación nasal con solución alcalina presentaron costras al final de los tres meses de terapia y en el seguimiento se observó la presencia de prácticamente todos los síntomas a las dos semanas posterior a la interrupción del tratamiento. En suma, el autor concluyó que los pacientes tratados con rifampicina tenían mejores resultados respecto a síntomas, signos y cambios histológicos en comparación con aquellos sometidos a inyecciones de extracto placentario e irrigación nasal con solución alcalina ${ }^{21}$.

En el año 2014, Friji presentó un estudio de 5 pacientes a los que se les realizó un implante nasal de lipoaspirado autólogo. Las zonas escogidas como donante fueron el área medial de la rodilla, región abdominal y troncantérica. Todos los pacientes refirieron mejoría en los síntomas dentro de las tres semanas posteriores a la intervención. A seis meses de la cirugía, todos refirieron desaparición total de costras nasales, con presencia de mucosa nasal normal y sin signos de atrofia. No se reportaron eventos adversos y las puntuaciones SNOT-20 mejoraron a los seis meses posoperatorios. El tiempo de aclaramiento mucociliar nasal fue reducido significativamente después de la operación (960 segundos) en comparación con el valor preoperatorio (1.995 segundos) 22 . En 2018, Park publicó el caso de una mujer de 63 años con rinitis atrófica primaria sin respuesta a la terapia médica en la que se realizó una reconstrucción del cornete inferior con cartílago costal autólogo. No presentó complicaciones y un mes después de la cirugía, sus síntomas mejoraron de manera significativa. A los dos años de seguimiento, su puntaje en la escala SNOT-25 fue de 6, con una puntuación inicial de $108^{23}$.

\section{Turbinectomía media y cirugía endoscópica nasal}

En 1973, Ssali propuso una nueva intervención quirúrgica para el tratamiento de la rinitis atrófica, la turbinectomía media. Se basaba en la hipótesis de que el cornete medio era el responsable de la obstrucción del drenaje del seno etmoidal con la consecuente estasia de secreciones y posterior sobreinfección. Luego se generaba inflamación crónica y metaplasia, lo que llevaba finalmente a la formación de costras y atrofia tanto de la mucosa como del hueso subyacente. En dicho estudio, la turbinectomía media fue realizada en un total de 35 pacientes, con un periodo de seguimiento de 24 meses. El $91 \%$ presentó resultados favorables, con desaparición de las costras y del mal olor y recuperación parcial del olfato. Los pacientes restantes $(9 \%)$ presentaron sólo una ligera disminución de la formación de costras. No se reportan complicaciones en los sujetos de estudio ${ }^{24}$.

En 1986 Stammberger introdujo las bases de la cirugía endoscópica funcional, con lo que buscaba inicialmente restaurar las alteraciones de la función tubaria y seno etmoidal ${ }^{25}$. En 1998, Fang publicó un ensayo clínico donde se realizó cirugía endoscópica a 14 pacientes con diagnóstico de rinitis atrófica. La intervención se llevó a cabo siguiendo las técnicas de Stammberger junto con turbinectomía media. En los resultados se evaluó la presencia de síntomas, imágenes, tiempo de aclaramiento de sacarina, y cultivos bacterianos, antes y dos años después de la cirugía. Un grupo de pacientes presentó buena recuperación mostrando cavidades nasales claras y normalización del sistema de transporte mucociliar. Sin embargo, dichos pacientes eran aquellos en los que existía evidencia de infección (imágenes sugerentes, y cultivo positivo para Klebsiella ozaenae), por lo que se concluyó que la cirugía endoscópica tenía buenos resultados en pacientes donde existía 
concomitancia con síntomas y signos de sinusitis crónica. Por el contrario, aquellos con escasos signos infecciosos tenían peores resultados ${ }^{10}$. En el mismo año, Jiang dio a conocer un estudio realizado con un total de 29 pacientes con rinitis atrófica, quienes fueron sometidos a cirugía endoscópica sinusal que incluía turbinectomía media junto con uso de aminoglicósidos en el posoperatorio. Vale la pena destacar que en dicho estudio se realizó antroscopía en 34 senos maxilares, observándose grados variables de inflamación en el $55 \%$ y secreción mucopurulenta en $27 \%$ de ellos. El manejo propuesto reportó eficacia de 92,6\%, tanto en recuperación parcial como total a 18 meses de seguimiento ${ }^{26}$.

\section{OTRAS TÉCNICAS NO QUIRÚRGICAS}

\section{Bloqueo neural}

En 1961, considerando la isquemia local como la causa principal de la patogénesis la rinitis atrófica, Bahl propuso el bloqueo del ganglio estrellado con el objetivo de mejorar el suministro vascular a la mucosa nasal. Un grupo de 25 pacientes fueron sometidos al procedimiento a través de la utilización de lidocaína junto con un vasoconstrictor por aproximadamente 40 días consecutivos. Paralelamente un grupo control de 15 pacientes fue sometido a manejo con irrigación nasal y aplicaciones nasales de glucosa al $25 \%$. Los resultados fueron evaluados en función de la desaparición de las costras nasales y el mal olor. En el grupo con bloqueo ganglionar se observó alivio sintomático en 20 casos, mientras que los 5 casos restantes no mostraron mejoría. En la serie de control, 7 casos mostraron leve disminución de los síntomas. Ocurrió recurrencia una vez finalizada la terapia dentro de uno a dos días en la serie de control y en tres a cuatro días en la serie sometida a bloqueo simpático. Con estos resultados, se concluyó que éste último, tenía solo un efecto beneficioso transitorio ${ }^{18}$.

\section{Administración sistémica de extracto placentario}

En 1957 Filtov estudió el supuesto rol de la placenta como estimulador de la regeneración de tejidos en diversas patologías. Basado en lo anterior, Karnik en 1971 puso a prueba el efecto de la inyección intramuscular de extracto placentario en 35 casos de rinitis atrófica. Se administró un ciclo completo de 20 inyecciones a cada paciente en días alternos. En el ámbito clínico 20 pacientes estuvieron libres de síntomas por dos años, sin considerar anosmia e hiposmia. Por su parte, 12 pacientes presentaron costras mínimas, mientras que 3 casos no presentaron mejoría. En suma, 80\% de los pacientes presentó mejora de sus síntomas mientras que el otro $20 \%$ presentó recurrencia de la enfermedad en 2 años de seguimiento ${ }^{12}$.

\section{Obturadores y stents nasales}

En 1998 se describió una técnica no quirúrgica que buscaba cerrar el vestíbulo nasal en casos de rinitis atrófica adquirida de forma secundaria. Consistía en ocluir las fosas nasales con un obturador hecho de dimetilpolisiloxano. Al ser un método no invasivo, estaba específicamente indicado en el tratamiento de casos de rinitis atrófica, en los que estaba contraindicado cualquier tratamiento quirúrgico. La experiencia en los cuatro pacientes donde se realizó fue alentadora y no se describieron efectos adversos asociados ${ }^{27}$. Siguiendo la misma línea, en el año 2008, Kumar publicó un artículo acerca del uso de stents nasal de acrílicos como tratamiento sintomático para la rinitis atrófica. Entre sus ventajas se mencionaba el hecho de ser una técnica no invasiva, inocua para los tejidos, costo-efectiva, fácil de usar y tolerable desde el punto de vista estético. El método fue utilizado en una mujer de 30 años que había sido sometida a cirugía de Young con malos resultados, y a la cual se habían agregado a sus síntomas la presencia de respiración bucal e hipernasalidad. Una vez confeccionada la prótesis, se debió realizar un pequeño agujero en cada molde nasal para permitir el flujo de aire, ya que la paciente presentó mala tolerancia a la oclusión bilateral total. A los dos meses de seguimiento, presentó disminución de la obstrucción nasal y cefalea, observándose cavidades nasales limpias y ausencia de costras y mal olor. A los 10 meses se describió que se encontraba libre de enfermedad ${ }^{28}$.

Con respecto al nivel de evidencia de cada una de las intervenciones mencionadas, sólo se 
encontró una revisión sistemática publicada por el grupo Cochrane en el año 2012 que informó que no hay evidencia a partir de ensayos controlados aleatorizados respecto a los beneficios o riesgos a largo plazo de las diferentes modalidades de tratamiento para la rinitis atrófica ${ }^{10}$. En cuanto a la rinitis atrófica unilateral, es importante mencionar que se ha asociado con la desviación septal, lo que causa una alteración del ciclo nasal que produce las alteraciones patológicas características de la rinitis atrófica. Por lo anterior se ha descrito a la septoplastía como una opción terapéutica².

\section{DISCUSIÓN}

A través de la historia y junto con el desarrollo tecnológicose han propuesto múltiples intervenciones quirúrgicas como método de resolución de la rinitis atrófica. Sin embargo, el bajo número de pacientes de las series, sumado a su escaso poder estadístico, hace que los resultados de los estudios revisados no sean válidos como alternativas terapéuticas para esta enfermedad. Por otra parte, en la mayoría de las publicaciones no existe una descripción detallada de la técnica quirúrgica ni reporte de las complicaciones $\mathrm{y} / 0$ eventos adversos observados en las intervenciones realizadas. Adicionalmente, los períodos de seguimiento acotados a pocos meses 0 años hacen difícil extrapolar los beneficios y la ausencia de complicaciones a la totalidad de los pacientes. No existe evidencia científica a partir de ensayos controlados aleatorizados respecto a los

\section{BIBLIOGRAFÍA}

1. deShazo RD, Stringer SP. Atrophic rhinosinusitis: progress toward explanation of an unsolved medical mystery. Curr Opin Allergy Clin Immunol 2011; 11: 1-7. doi:10.1097/ ACl.0b013e328342333e.

2. Cabrera N, Ruz S, Valdés C. Rinitis Atrófica. Rev Otorrinolaringol Cir Cabeza Cuello 2013; 73: 276-80.

3. Bist SS, Bisht M, Purohit JP, Saxena R. Study of histopathological changes in primary atrophic rhinitis. ISRN Otolaryngol 2011; 2011: 269479. doi:10.5402/2011/269479. beneficios o riesgos a largo plazo de las diferentes modalidades de tratamiento, lo que plantea un conflicto ético importante, ya que se hace complejo plantear estudios que sometan a los pacientes a las técnicas descritas. Finalmente, parece razonable el uso de cirugía endoscópica funcional en aquellos casos de rinitis atrófica que no respondan a tratamiento conservador y más aún en los que exista algún grado de inflamación crónica de las cavidades paranasales.

\section{CONCLUSIÓN}

La rinitis atrófica es una enfermedad crónica y progresiva de la mucosa nasal cuyo tratamiento no es curativo, por lo que se han desarrollado distintas alternativas terapéuticas, siendo el tratamiento conservador el más ampliamente utilizado hasta la fecha. A pesar de que no existe evidencia publicada acerca de la utilidad y el momento adecuado para la toma de cultivos, parece pertinente considerar su obtención en pacientes con clínica persistente y mala respuesta al tratamiento médico. Con respecto al tratamiento quirúrgico, si bien la mayoría de las técnicas descritas ya no se utilizan en la actualidad, es importante conocerlas debido a que aún existen pacientes que fueron sometidos a ellas, pudiendo presentar algunas de sus complicaciones $\mathrm{y} / 0$ efectos adversos. Finalmente, es importante mencionar que el correcto diagnóstico etiológico de la rinitis atrófica permitirá entregar al paciente una mejor alternativa terapéutica.

4. Dutt SN, Kameswaran M. The aetiology and management of atrophic rhinitis. J Laryngol Otol 2005; 119: 843-52. doi:10.1258/002221505774783377.

5. García G, Bailie N, Martins D y cols. Atrophic rhinitis: a CFD study of air conditioning in the nasal cavity. J Appl Physio/2007; 103: 1082-92.

6. Banks T, Gada S. Atrophic rhinitis. Allergy Asthma Proc 2013; 34: 185-7.

7. Shah K, Guarderas J, Krishnaswamy G. Empty nose syndrome and atrophic rhinitis. Ann Allergy Asthma Immunol 2016; 117; 217-20.

8. Chhabra N, Houser S. The Diagnosis and Management of Empty Nose Syndrome. Otolaryngol Clin North Am 2009; 42: 311-30. 
9. Hildenbrand T, Rainer KW, Brehmer D. Rhinitis sicca, dry nose and atrophic rhinitis: a review of the literature. Eur Arch Otorhinolaryngol 2010; 268: 17-26.

10. Mishra A, Kawatra R, Gola M. Interventions for atrophic rhinitis. Cochrane Database Syst Rev 2012; 2: CD008280. doi:10.1002/14651858. CD008280.pub2.

11. Young A. Closure of the nostrils in atrophic rhinitis. J Laryngol Otol 1967; 81: 515-24. doi:10.1017/s0022215100067426.

12. Sinha SN, Sardana DS, Rajvanshi VS. A nine years' review of 273 cases of atrophic rhinitis and its management. J Laryngol Otol 1977; 91: 591600. doi:10.1017/s0022215100084097.

13. Gadre KC, Bhargava KB, Pradhan RY, Lodaya JD, Ingle MV. Closure of the nostrils (Young's operation) in atrophic rhinitis. J Laryngol Otol 1971; 85: 711-4. doi:10.1017/s0022215100073977.

14. Elwany S. Ultrastructural observations on primary atrophic rhinitis. Effect of partial closure of the nostril. ORL J Otorhinolaryngol Relat Spec 1988; 50: 389-96. doi:10.1159/000276019.

15. Poddar SK, Jagade MV. Modification of modified young's operation in the management of primary atrophic rhinitis. Indian J Otolaryngol Head Neck Surg 2001; 53: 252-4. doi:10.1007/BF03028570.

16. SHARAN R. Transplantation of maxillary sinus mucosa in atrophic rhinitis. Indian J Otolaryngol 1978; 30: 14-6.

17. Proud G. Acrylic resin implant for atrophic rhinitis (preliminary report). Laryngoscope 1974; 57: 256-62.

18. SAUNDERS WH. Atrophic rhinitis; results of surgical treatment. AMA Arch Otolaryngol 1958; 68: 342-5.

19. Goldenberg D, Danino J, Netzer A, Joachims HZ. Plastipore implants in the surgical treatment of atrophic rhinitis: technique and results. Otolaryngol Head Neck Surg 2000; 122: 794-7. doi:10.1016/s0194-5998(00)70003-9.

20. HOUSER SM. Surgical treatment for empty nose syndrome. Arch Otolaryngol Head Neck Surg 2007; 133: 858-63. doi:10.1001/archotol.133.9.858.

21. Jaswal A, Kumar K, Sikder B et al. Novel treatment of atrophic rhinitis: early results. Eur Arch Otorhinolaryngol 2008; 265: 1211-17.

22. FriJu MT, Gopalakrishnan S, Verma SK y cols. New regenerative approach to Atrophic Rhinitis using autologous lipoaspirate transfer and platelet rich plasma in five patients: Our Experience. Clin Otolaryngol 2014; 39: 289-92.

23. Park MJ, Jang YJ. Successful management of primary atrophic rhinitis by turbinate reconstruction using autologous costal cartilage. Auris Nasus Larynx 2018; 45: 613-6.

24. Ssal CL. Atrophic rhinitis. A new curative surgical treatment (middle turbinectomy). J Laryngol Otol 1973; 87: 397-403.

25. Stammberger H. An endoscopic study of tubal function and the diseased ethmoid sinus. Arch Otorhinolaryngol 1986; 243: 254-9.

26. Jiang RS, Hsu CY, Chen CC, Jan YJ, Jang JW. Endoscopic sinus surgery and postoperative intravenous aminoglycosides in the treatment of atrophic rhinitis. Am J Rhinol 1998; 12: 325-33. doi:10.2500/105065898780182480.

27. Lobo CJ, Hartley C, Farrington WT. Closure of the nasal vestibule in atrophic rhinitis--a new non-surgical technique. J Laryngol Otol 1998; 112: 543-6.

28. Kumar NS, Chandra TS. Prosthetic management of atrophic rhinitis. Indian J Otolaryngol Head Neck Surg 2008; 60: 379-81. doi:10.1007/ s12070-008-0120-z. 